Zhen Wang", Fu-Hou Lei ${ }^{\# \star}$, Wen Li ${ }^{\# \star}$, Peng-Fei Li\#, Ting Wang, Li-Ting Qin, Ge-Ge Cheng, Yao Sun, Yu-Yu E, Wen-Bo Xie and Jie Deng

\title{
Preparation of rosin-based polymer microspheres as a stationary phase in high-performance liquid chromatography to separate polycyclic aromatic hydrocarbons and alkaloids
}

https://doi.org/10.1515/epoly-2019-0029

Received November 12, 2018; accepted December 17, 2018.

\begin{abstract}
Acrylpimaric acid ethylene glycol acrylate was used as the cross-linking agent to prepare rosin-based polymer microspheres through membrane emulsification-microsuspension polymerization. The prepared microspheres were used as the stationary phase in highperformance liquid chromatography for the separation of polycyclic aromatic hydrocarbons (PAHs) and two analogues of alkaloids. Five PAHs (benzene, naphthalene, fluorene, pyrene, and benzo(e)pyrene) were well separated in the rosin-based polymer column with the resolution (Rs) of $6.088 \pm 0.006,5.759 \pm 0.017,7.019 \pm 0.020$, and $8.584 \pm 0.063$. A linear relationship was observed for the carbon numbers of PAHs and $\operatorname{lnK}$ as obtained with the equation of $\ln \mathrm{k}=0.22 n_{c}-2.45, \mathrm{R}^{2}=0.996$. The rosinbased polymer columns were also employed to separate
\end{abstract}

\footnotetext{
* Corresponding author: Fu-Hou Lei and Wen Li, College of Chemistry and Chemical Engineering, Guangxi University for nationalities, Nanning 530006, China; Guangxi Key Laboratory of Chemistry and Engineering of Forest Products, Guangxi University for nationalities, Nanning 530006, China; College of Light Industry and Food Engineering, Guangxi University, Nanning 530004, China, e-mail: leifuhougxun@126.com (Fu-Hou Lei); gxliwen@st.gxu.edu.cn (Wen Li). Tel.: +86 07713267071.

Zhen Wang, Peng-Fei Li, Ting Wang and Li-Ting Qin, College of Chemistry and Chemical Engineering, Guangxi University for nationalities, Nanning 530006, China; Guangxi Key Laboratory of Chemistry and Engineering of Forest Products, Guangxi University for nationalities, Nanning 530006, China.

Ge-Ge Cheng, Yao Sun, Yu-Yu E and Wen-Bo Xie, College of Chemistry and Chemical Engineering, Guangxi University for nationalities, Nanning 530006, China.

Jie Deng, College of chemistry and Environmental Engineering, Hubei University for Nationalities, Enshi Tujia and Miao Autonomous Prefecture 445000, China.

"Zhen Wang, Fu-Hou Lei, Wen Li and Peng-Fei Li contributed equally to this work.
}

theophylline-caffeine and camptothecin-10-hydroxycamptothecin, and the good results were obtained with the Rs of $4.617 \pm 0.005$ and $2.245 \pm 0.049$, respectively.

Keywords: rosin-based stationary phase; alkaloids; polycyclic aromatic hydrocarbons; polymer; microspheres

\section{Introduction}

Polymer microspheres are polymeric materials or polymer composites with sizes ranging from nanometer to micrometer scale and spherical geometrical shapes (1-3). Polymer microspheres have wide applications in stationary phases, biological medicine and many other fields (4-7). Organic polymer stationary phases mainly include polysaccharide polymers, polystyrenes/ divinylbenzene polymers and polymethacrylate polymers (8-10). Due to the poor mechanical stability, when polysaccharide polymers and polymethacrylate polymers are used in high performance liquid phase, the particles are easy to collapse under high pressure (11). Polystyrenes/ divinylbenzene polymers can bear the high pressure and have a good adaptability for chromatographic purposes but may release residuals, which are harmful to health, when they are used for the separation and analysis of drugs $(12,13)$. As the ternary ring structure of rosin can be introduced into high molecular structure, rosin can enhance the mechanical strength of polymer and is an important resource to synthesize high-molecular stationary phase instead of divinylbenzene or styrene. In addition, rosin is biodegradable, non-toxic, and thus can be used as a raw material to separate and purify the effective ingredients of Chinese herbs (14).

Polycyclic aromatic hydrocarbons (PAHs) are carcinogenic pollutants that can be absorbed by crops via polluted water, air, or, soil (15). PAHs could be 
generated during the general extraction process of natural medicinal active components in a high temperature (15). Hence, detecting PAHs and removing them from natural medicines are important. Theophylline (THO)-caffeine (CAF) and camptothecin (CPT)-10-hydroxycamptothecin (HCPT) belong to the two sets of alkaloids with similar structures and are widely applied in clinical drugs $(16,17)$. Slight modification of the molecule configuration could lead to different pharmacology activities and applications. THO is used extensively to treat asthma, and CAF is often added to pain medications. Compared with CPT, HCPT holds up better in anticancer activity and has lower toxicity. Detecting and separating these two sets of alkaloids is of importance.

In our previous work (13), paclitaxel imprinted polymer microspheres (using ethylene glycol maleic rosinate acrylate (EGMRA) as cross-linking agent) were prepared by microsuspension polymerization. However, the particle size distribution of the microspheres prepared by microsuspension polymerization was relatively wide (1-50 $\mu \mathrm{m})$. As maleopimaric anhydride requires ring opening at high pressure to produce maleopimaric acid to introduce double bonds, the synthesis cost of EGMRA was higher compared with acrylpimaric acid ethylene glycol acrylate (AAEGA). In addition, the preparation process of molecularly imprinted polymer microspheres was rather complex, in which template molecules should be added and then removed after the formation of the microspheres. Template molecules are often expensive and difficult to be recycled, resulting in the high production cost of the polymers. Thus, it is very necessary and meaningful to develop the application field of non-imprinted polymer microspheres.
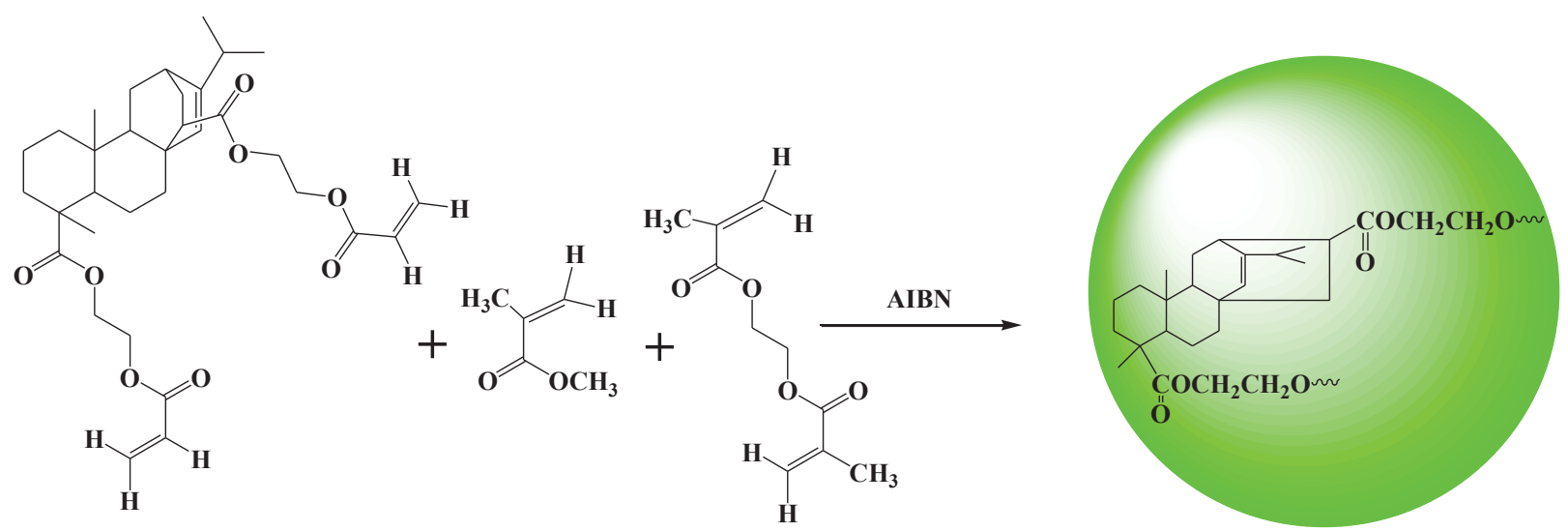
AAEGA
MMA
EGDMA
Rosin-based polymer microsphere

In this study, rosin-based polymer microspheres were prepared and used as the stationary phase in highperformance liquid chromatography (HPLC) to separate PAHs and alkaloids. The main objectives of this work are as follows: (1) preparation of polymer microspheres using acrylpimaric acid ethylene glycol acrylate (AAEGA) as the cross-linking agent through membrane emulsificationmicrosuspension polymerization; (2) preparation of the rosin-based polymer columns using the microspheres to separate PAHs, THO-CAF, and CPT-HCPT.

\section{Materials and methods}

\subsection{Experimental principles}

Figure 1 illustrates the principles and schematic of the preparation of rosin-based polymer microspheres. Methyl methacrylate (MMA), ethyleneglycol dimethacrylate (EGDMA) cross-linked with AAEGA effectively to form a macromolecule with network structure, and the threering phenanthrene skeleton structure with high rigidity in rosin was introduced into the polymer chain through free radical polymerization by using azodiisobutyronitrile (AIBN) as initiator. The AAEGA was provided by the Guangxi Key Laboratory of Forest Products Chemistry and Engineering (Nanning, China).

\subsection{Preparation of rosin-based polymer microspheres}

(i) MMA (10 mmol), EGDMA (4 mmol), AAEGA (10 mmol), chloroform (30 mL), cetanol, polypropylene glycol,

Figure 1: Principles of the synthesis of rosin-based polymer microspheres.

\section{Figure 1: Principles of the synthesis of rosin-based polymer microspheres.}


and AIBN were mixed together and ultrasonicated for $10 \mathrm{~min}$ as the oil phase (dispersion phase). The amounts of cetanol, polypropylene glycol, and AIBN were 5\%, $2 \%$, and $0.3 \%$ of the total mass of MAA, EGDMA, and AAEGA, respectively. (ii) Second, $40 \mathrm{~g}$ of the dispersion phase was added into the aqueous phase $(400 \mathrm{~mL})$ containing $2 \%(\mathrm{w} / \mathrm{w})$ polyvinyl alcohol (PVA) in deionized (DI) water. (iii) Crude oil-in-water emulsions were poured into the membrane emulsification setup (FM0210/500M, Senhui company, Beijing, China). The emulsions were homogenized by squeezing them through a membrane (SPG) with an average pore size of $8 \mu \mathrm{m}$ under the pressure of 1.0 bar and collected using a beaker. (iv) The emulsions (droplets) were heated to $75^{\circ} \mathrm{C}$ and maintained for $2 \mathrm{~h}$, raised to $80^{\circ} \mathrm{C}$ for $2 \mathrm{~h}$, and aged at $95^{\circ} \mathrm{C}$ for $2 \mathrm{~h}$. The copolymers were then treated through sedimentation and centrifugation. (v) Finally, porogens, unreacted monomers and assistant agents were removed through Soxhlet extraction with acetone, ethyl acetate, and ethyl alcohol, respectively. The final products (microspheres) were obtained after washing with DI water for removing PVA.

\subsection{Characterization}

Scanning electron microscopy (SEM; SUPPRA 55 Sapphire, Carl Zeiss, Germany) was conducted to investigate the sample's morphology. Particle sizes and distributions of microspheres were measured by laser granulometry (Mastersizer 3000, Malvern, UK). Specific surface area and porosity were examined through $\mathrm{N}_{2}$ adsorption experiments by using a surface area and pore size analyzer (ASAP 2020, Micromeritics, Atlanta, USA) at $77 \mathrm{~K}$. Surface areas were calculated by the Brunauer-Emmett-Teller (BET) equations. Pore volume, average pore size, and pore size distribution were calculated using the Barrett-Joyner-Halenda equations. The thermal stabilities of the microspheres were characterized by thermogravimetric analysis (TGA; STA449F3, Netzsch-Gerätebau GmbH, Germany). The samples (2-4 $\mathrm{mg}$ ) were placed in a standard crucible $\left(\mathrm{Al}_{2} \mathrm{O}_{3}\right)$ and heated under nitrogen from $30^{\circ} \mathrm{C}$ to $800^{\circ} \mathrm{C}$ at a rate of $10^{\circ} \mathrm{C} \mathrm{min}{ }^{-1}$ to obtain thermogravimetric and differential thermogravimetric curves.

\subsection{Chromatographic evaluation of polymers}

The copolymers were packed into a stainless-steel chromatographic column $(250 \mathrm{~mm} \times 4.6 \mathrm{~mm}$ i.d. $)$ with the wet method of packing column by using a slurry packer
(CP-24, Scientific Systems company, Science Park Road State College, PA, USA) under the pressure of 3250 psi with chloroform-methanol $(2.6: 1, \mathrm{v} / \mathrm{v})$ as the slurry solvent. The prepared rosin-based polymer column was connected to an HPLC (LC-6AD, Shimadzu, Japan) system and flushed with $4 \mathrm{~L}$ of methanol at $0.1 \mathrm{~mL} / \mathrm{min}$.

Chromatographic experiments were performed on an HPLC (LC-6AD, Shimadzu, Japan) system with a UV detector (Shimadzu, Japan). Unless otherwise stated, separations were conducted at $30^{\circ} \mathrm{C}$ using methanol as mobile phase at a flow rate of $0.3 \mathrm{~mL} / \mathrm{min}$. In brief, $20 \mu \mathrm{L}$ of each sample was injected, and the wavelength of the UV detector was set at $254 \mathrm{~nm}$. $R_{S}$ the resolution, is expressed as follows:

$$
R \mathrm{~s}=\frac{2\left(\mathrm{t}_{\mathrm{R} 2}-t_{R 1}\right)}{W_{1}+W_{2}}
$$

Where $t_{R 1}$ and $t_{R 2}$ is the is the retention time of Peak 1 and Peak 2, respectively, and $\mathrm{W}_{1}$ and $\mathrm{W}_{2}$ is the peak width of Peak 1 and Peak 2, respectively.

The mixed solution of PAHs was prepared, in which $1 \mathrm{~L}$ of solution (methanol as the solvent) contains benzene, naphthalene, fluorine, pyrene, and benzo(e) pyrene at $0.05,0.005,2.5 \times 10^{-4}, 2.5 \times 10^{-3}$, and $2.5 \times 10^{-3} \mathrm{~mol}$, respectively. The mixed solution of THO and CAF (CPT and HCPT) was prepared in methanol with concentrations of $1.25 \times 10^{-4} \mathrm{~mol} \mathrm{~L}^{-1}$.

\section{Results and discussion}

\subsection{Characterization of rosin-based polymer microspheres}

In Figure 2a, microspheres with a narrow pore distribution exhibit unimodal particle size distribution profiles ranging from $1 \mu \mathrm{m}$ to $10 \mu \mathrm{m}$, which meets the requirement for particle sizes of the liquid chromatographic stationary phase. The thermal weight loss of the polymer microspheres was characterized by TGA, and the results are presented in Figure $2 \mathrm{~b}$. The decomposition temperature for polymer microspheres was about $280^{\circ} \mathrm{C}$. The temperature at the highest decomposition rates was about $422^{\circ} \mathrm{C}$, and decomposition was completed at about $480^{\circ} \mathrm{C}$. Thus, TGA demonstrated that the microspheres possessed good thermal stability. Figure $2 \mathrm{c}$ shows that the microspheres displayed typical-IV isotherms with well-defined $\mathrm{H}_{1}$ hysteresis loops, implying a uniform mesoporous structure (Figure 2d) containing cylindrical 

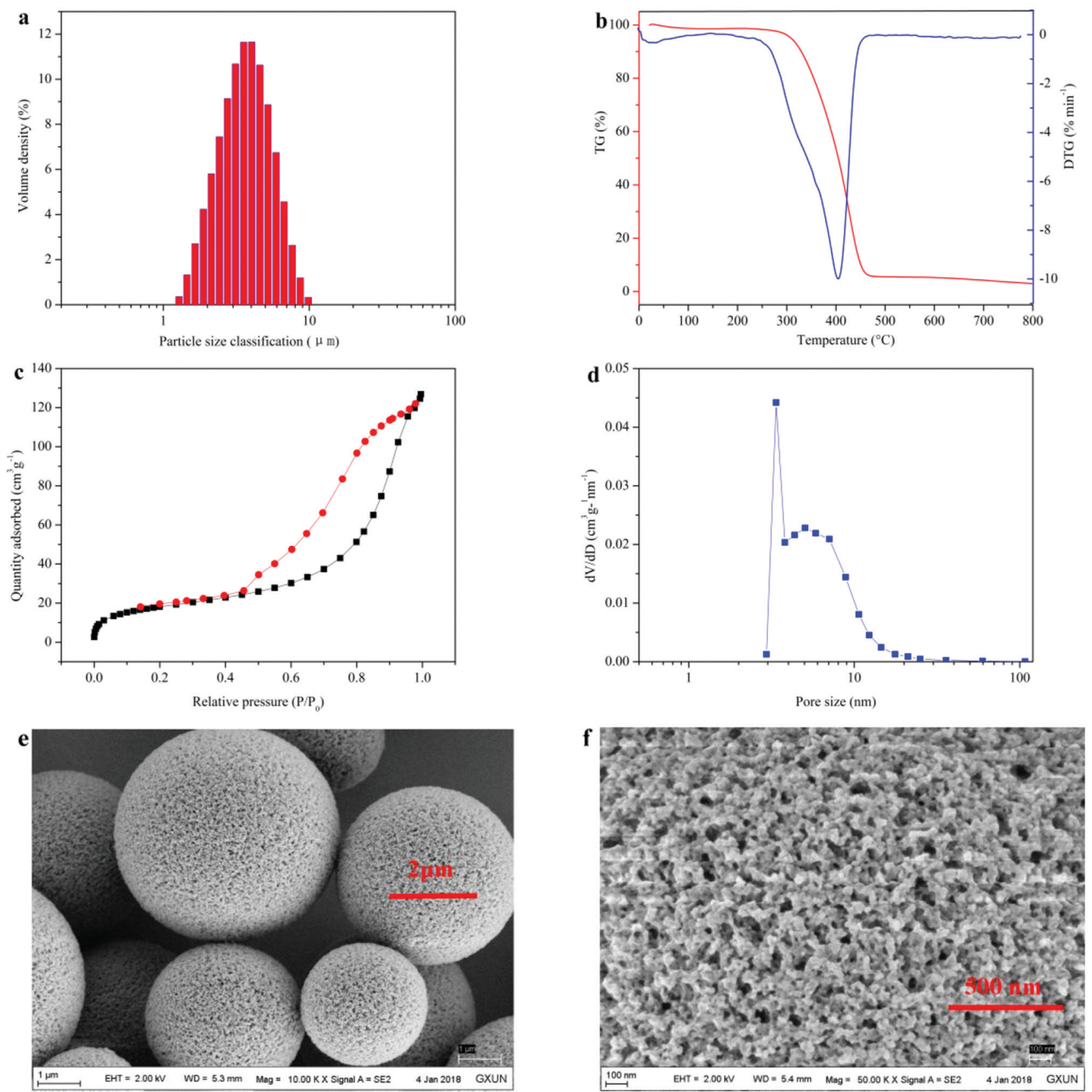

Figure 2: Characterization of microspheres. (a) Particle size distribution of microspheres. (b) Weight loss of microspheres. (c) $\mathrm{N}_{2}$ adsorption-desorption isotherm of microspheres. (d) Pore size distribution of microspheres. (e) SEM image of microspheres which was magnified to one million times. (f) SEM image of microspheres which was magnified to five millions times.

pores. The BET surface area, pore volume, average pore diameter, and average pore size of the microspheres are $67.4723 \mathrm{~m}^{2} / \mathrm{g}, 0.2026 \mathrm{~cm}^{3} / \mathrm{g}$, and $6.8192 \mathrm{~nm}$, respectively. The SEM micrographs of the microspheres magnified to 1 and 5 million times are shown in Figures 2e and 2f. As shown in Figure 2e, the rosin-based polymers are regular spheres with a rough and porous surface, and the pores in the microspheres have formed a 3D network (Figure 2f).

\subsection{Chromatographic characterization of polymers}

Figure 3a shows that five PAHs were well separated in the rosin-based polymer column with the resolution (Rs) (the two components which elute closest to each other) of $6.088 \pm 0.006,5.759 \pm 0.017,7.019 \pm 0.020$, and $8.584 \pm 0.063$. Figure $3 \mathrm{~b}$ shows a linear relationship 

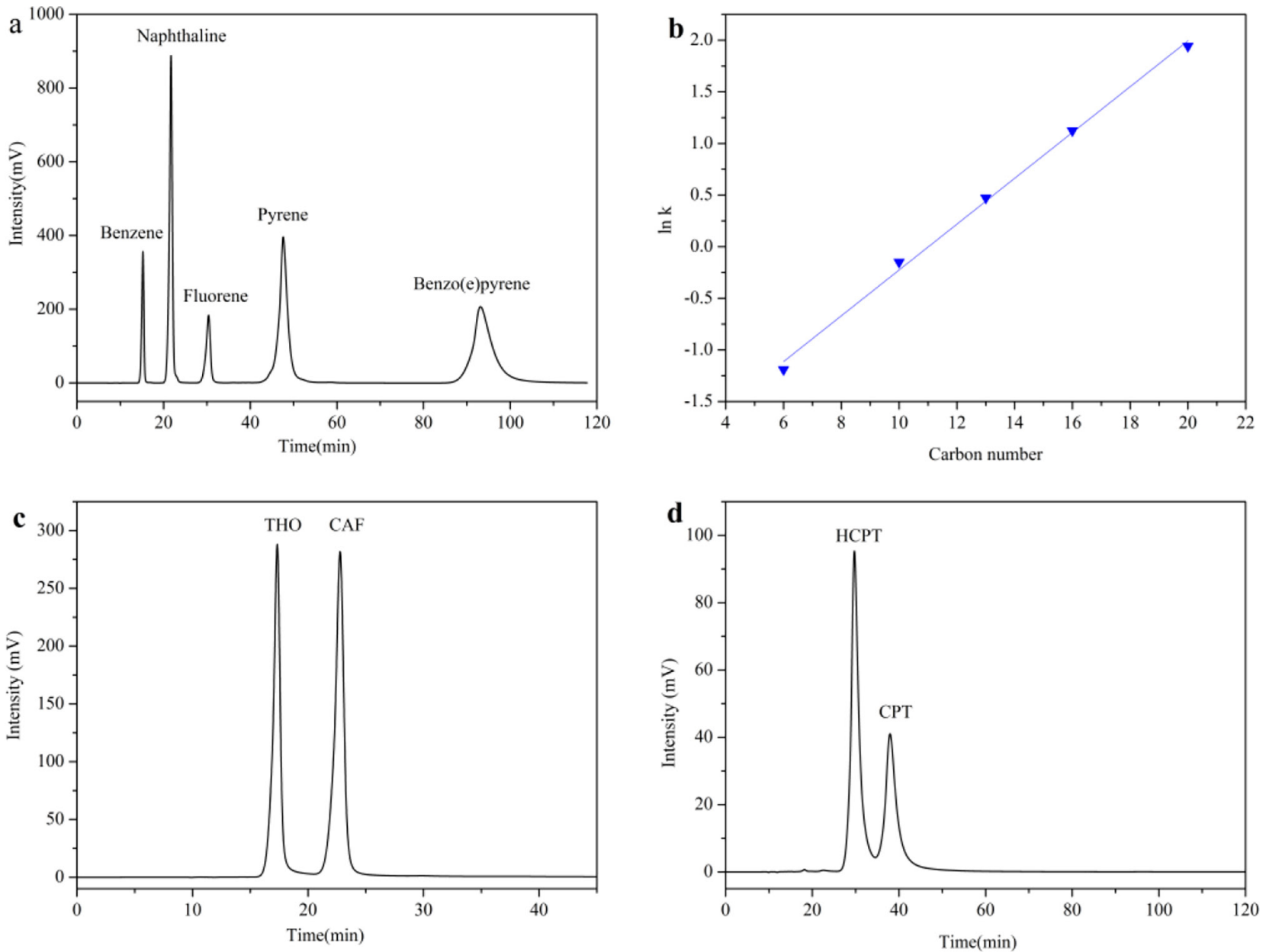

Figure 3: (a) Separation of mixed samples of PAHs in the chromatographic column. (b) The carbon number rule plots obtained by plotting In $k$ and $n_{\text {. }}$ (c) Separation of mixed samples of THO and CAF in the chromatographic column. (d) Separation of mixed samples of CPT and HCPT in the chromatographic column.

between the carbon numbers of PAHs and the $\ln \mathrm{k}$, which followed the equation of the carbon number rule:

$$
\ln \mathrm{k}=a n_{c}+b
$$

Where $\mathrm{k}$ is the retention factor, and $n_{c}$ is the homologue carbon atom number (18). This linear fitting equation is as follows:

$$
\ln \mathrm{k}=0.22 \mathrm{n}_{\mathrm{c}}-2.45, \mathrm{R}^{2}=0.996 .
$$

Basing on the equation and Figure 3a, we can discover information as follows: (1) According to the equation, we can infer the retention value of other PHAs. (2) All of the five PAHs can enter into the hole interior of polymers; otherwise, the relationship shows an fold line (19). (3) When the sample enters the hole interior, the mass transfer resistance of the molecules changes according to molecular weights. The diffusion rate quickens when the molecular weight decreases (20). (4) The rosin-based polymer microspheres can be used in the separation of organic compounds with similar structure but different molecular sizes.

As shown in Figure 3c, THO and CAF obtained a good separation ( $R s=4.6 \pm 0.005)$. The structure of the CAF has one more methyl group than THO (Figure 4), which increases the mass transfer resistance, leading to the prolonged retention time. Moreover, the N-H bonds of the THO can form hydrogen with the methanol, which also helps the separation.

As shown in Figure 3d, the Rs of CPT and HCPT is $2.245 \pm 0.049$. CPT and HCPT belong to polycyclic alkaloid; hence, their retention times were longer than those of THO and CAF. The molecular structure of HCPT is different than that of CPT due to a hydroxyl (Figure 4), and the extra hydroxyl increased the polarity of HCPT. When using 
<smiles>CCC1(O)C(=O)OCc2c1cc1n(c2=O)Cc2cc3ccccc3nc2-1</smiles>

Camptothecin (CPT)<smiles>CCC1(O)C(=O)OCc2c1cc1n(c2=O)Cc2cc3cc(O)ccc3nc2-1</smiles>

10-hydroxycamptothecin (HCPT)<smiles>C=C1N(C)C(=O)c2nc[nH]c2N1C</smiles>

Theophylline (THO)<smiles>C=C1N(C)C(=O)c2c(ncn2C)N1C</smiles>

Caffeine (CAF)

Figure 4: Structures of CPT, HCPT, THO and CAF.

methyl alcohol as the mobile phase, the influence of the polarity of the sample molecules plays a major role in the chromatographic separation. As a result, the retention time of HCPT was shorter than that of CPT.

\section{Conclusion}

In this study, AAEGA was used as the cross-linking agent to prepare rosin-based polymer microspheres through membrane emulsification-microsuspension polymerization. Rosin-based polymer columns were prepared by the slurry method. Finally, the rosin-based polymer columns were applied to separate PAHs, THOCAF and CPT-HCPT.

(1) Five PAHs were well separated in the rosin-based polymer column with the Rs of $6.088 \pm 0.006$, $5.759 \pm 0.017,7.019 \pm 0.020$, and $8.584 \pm 0.063$.

(2) The rosin-based polymer column could thoroughly separate THO-CAF and CPT-HCPT with Rs of $4.617 \pm 0.005$ and $2.245 \pm 0.049$, respectively.

(3) The non-toxic and environmentally friendly rosinbased polymer microspheres show potential to be used as packing material of preparative column which can be applied in the separation and purification of natural medications.

Acknowledgments: We thank the National Key Research and Development Program of China (Grant number: 2016YFD0600804), the National Natural Sciences Foundation of China (Grant number: 31700505), and the Base and Special Talent of Guangxi Science and

Technology (Grant number: 2017AD19029) for their financial support.

\section{References}

1. Eichler-Volf A., Kovalev A., Wedeking T., Gorb E.V., Xue L., You C., et al., Bioinspired monolithic polymer microsphere arrays as generically anti-adhesive surfaces. Bioinspir Biomim, 2016, 11(2), 25002-25013.

2. Sun D., Chen F., Gao Y., Wang Y., Synthesis of cross-linked polymer microspheres and pyrolysis conversion to polymerderived ceramics. Int J Appl Ceram Tec, 2017, 14(5), 992-998.

3. Lin M., Zhao Q., Dang S., Wang Y., Wang Y., Wang X., Preparation and properties of terpolymeric microspheres for deep profile control in oilfields. Mater Res Innov, 2015, 19(5), 574-579.

4. Valente J.F.A., Gaspar V.M., Antunes B.P., Countinho P., Correia I.J., Microencapsulated chitosan-dextran sulfate nanoparticles for controled delivery of bioactive molecules and cells in bone regeneration. Polymer, 2013, 54(1), 5-15.

5. Huang H., Wang H., Wu Y., Shi Y., Deng J.P., Chiral, crosslinked, and micron-sized spheres of substituted polyacetylene prepared by precipitation polymerization. Polymer, 2018, 139.

6. Gu W., Ting S.R.S., Stenzel M.H., Synthesis of pH-responsive and thiol-degradable hollow microspheres. Polymer, 2013, 54(3), 1010-1017.

7. Li P., Xu K., Tan Y., Lu C., Li Y., Wang P., A novel fabrication method of temperature-responsive poly(acrylamide) composite hydrogel with high mechanical strength. Polymer, 2013, 54(21), 5830-5838.

8. Lungfiel K., Seubert A., Varying the porous structure of polystyrene/divinylbenzene beads prepared by Ugelstads activated swelling technique and examining its reversed phase HPLC properties. J Chromatogr A, 2014, 1358, 117-127.

9. Vlakh E.G., Tennikova T.B., Applications of polymethacrylatebased monoliths in high-performance liquid chromatography. J Chromatogr A, 2009, 1216(13), 2637-2650.

10. Francotte E., Huynh D., Zhang T., Photochemically immobilized 4-methylbenzoyl cellulose as a powerful chiral stationary phase for enantioselective chromatography. Molecules, 2016, 21(12), 1740.

11. Qu J.B., Huang Y.D., Jing G.L., Liu J.G., Zhou W.Q., Zhu H., et al., A novel matrix derivatized from hydrophilic gigaporous polystyrene-based microspheres for high-speed immobilizedmetal affinity chromatography. J Chromatogr B, 2011, 879(15), 1043-1048.

12. Lungfiel J., Seubert A., Varying the porous structure of polystyrene/divinylbenzene beads prepared by Ugelstads activated swelling technique and examining its reversed phase HPLC properties. J Chromatogr A, 2014, 1358, 117-127.

13. Li P., Wang T., Lei F., Peng X., Wang H., Qin L., et al., Preparation and evaluation of paclitaxel-imprinted polymers with a rosin-based crosslinker as the stationary phase in high-performance liquid chromatography. J Chromatogr A, 2017, 1502, 30-37.

14. Li P., Wang T., Lei F., Tang P.P., Tan X.C., Liu Z.G., et al., Rosin-based molecularly imprinted polymers as the stationary phase in high-performance liquid chromatography for selective 
separation of berberine hydrochloride. Polym Int, 2014, 63(9), 1699-1706.

15. Kim K.H., Jahan S.A., Kabir E., Brown R.J.C., A review of airborne polycyclic aromatic hydrocarbons (PAHs) and their human health effects. Environ Int, 2013, 60(5), 71-80.

16. Jeong J., Kim H.S., Song E.S., Choi Y.Y., Comparison between Caffeine and Theophylline Therapy for Apnea of Prematurity. Neonatal Medicine, 2015, 22(1), 14-20.

17. Hong M.H., Zhu S.J., Jiang Y.Y., Tang G.T., Sun C., Fang C., et al., Novel anti-tumor strategy: PEG-hydroxycamptothecin conjugate loaded transferrin-PEG-nanoparticles. J Control Release, 2010, 141(1), 22-29.
18. Zhang X.X., Liu Y.Q., Yu H., Zhang R.Q., Rapid and simultaneous determination of piperidinium and pyrrolidinium ionic liquid cations by ion pair chromatography coupled with direct conductivity detection. Chinese Chem Lett, 2017, 28(1), 126-130.

19. Jiao Q.C., Liu Q., Chen Y.Z., Investigations of retention rule in reversed-phase liquid chromatography. Acta Chim Sinica, 1998, 56, 68-76 (in Chinese which English abstract).

20. Deng L.R., Hu X.Z., Wu Q.H., Meng Q.C., Li Y.L., Zhang Y.L., Study of dependence of liquid chromatographic performance upon pore structure of porous polydivinylbenzene microspheres. Sci China Chem, 1982, 25(9), 905-915. 